\begin{tabular}{|c|c|c|c|c|c|c|c|c|c|}
\hline \multirow{2}{*}{$\begin{array}{c}\text { Konstanten } \\
\text { der Butterfette }\end{array}$} & \multicolumn{6}{|c|}{ Butter bei Stallfütterung } & \multicolumn{3}{|c|}{ Butter bei Weidegang } \\
\hline & A & B & $\mathrm{C}$ & $\mathrm{D}$ & E & $\mathrm{F}$ & G & $\mathrm{H}$ & I \\
\hline bei $40^{\circ}$ & - & 46,9 & 47,0 & 46,3 & 46,6 & 46,6 & 47,2 & 46,8 & 47,0 \\
\hline Refrak- Spezial jW & $+3,7$ & $+3,7$ & $+4,0$ & $+3,0$ & $+3,2$ & $+3,1$ & $+4,0$ & $+3,5$ & $+3,7$ \\
\hline thermometer $\mathrm{S}$ & - & - & - & - & - & - & $+2,2$ & $+1,7$ & $+1,9$ \\
\hline Reichert-MeiBl'sche Zahl & 19,3 & 17,5 & 16,8 & 17,8 & 15,4 & 17,6 & 17,6 & 23,6 & 22,2 \\
\hline Polenske'sche Zahl . . . & - & 1,55 & 1,7 & 0,8 & 0,7 & 0,85 & 1,2 & 1,6 & 1,2 \\
\hline Verseifungszahl . . . . . & 213,0 & 209,3 & 206,7 & 206,7 & 206,9 & 205,5 & 209,0 & 215,3 & 212,4 \\
\hline $\begin{array}{l}\text { Molekulargewicht der nicht- } \\
\text { flüchtigen unlöslichen Fett- }\end{array}$ & & & & & & & & & \\
\hline 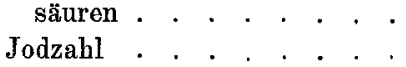 & - & $\begin{array}{r}271,0 \\
42,0\end{array}$ & $\begin{array}{r}271,0 \\
44,4\end{array}$ & $\begin{array}{r}272,2 \\
39,0\end{array}$ & $\begin{array}{r}270,7 \\
40,7\end{array}$ & $\begin{array}{r}272,2 \\
39,7\end{array}$ & $\begin{array}{r}269,6 \\
47,3\end{array}$ & $\begin{array}{r}268,3 \\
44,7\end{array}$ & $\begin{array}{r}267,0 \\
46,3\end{array}$ \\
\hline Schmelzpunkt . & - & 41,5 & 39,2 & 44,2 & 42,5 & 45,3 & 35,5 & 35,7 & 36,0 \\
\hline Erstarrungspunkt. . . & - & 27,8 & 27,9 & 31,4 & 28,2 & 30,9 & 24,4 & 22,4 & 22,6 \\
\hline
\end{tabular}

\title{
Über die Beziehungen zwischen der Zusammensetzung von Fruchtsaftaschen und ihrer Alkalität.
}

\section{Von}

\section{A. Beythien.}

Mitteilung aus dem Chemischen Untersuchungsamte der Stadt Dresden.

Die Aufsehen erregende Veröffentlichung, durch welche Herr Dr. F. EversDüsseldorf ${ }^{1}$ ) im Vorjahre versuchte, die Analyse und Begutachtung des Himbeersyrups in neue Bahnen zu lenken, hat in der wissenschaftlichen Literatur keine Anerkennung, hingegen von zahlreichen Fachgenossen wie Spaeth ${ }^{8}$ ), Jucken ack und Pasternack ${ }^{3}$ ), Lührig ${ }^{4}$ ), Buttenberg ${ }^{5}$ ), Lepère ${ }^{6}$ ), Matthes ${ }^{7}$ ) so entschiedenen Widerspruch gefunden, daß sie für die Kreise der Nahrungsmittelchemiker als abgetan zu gelten hat. Wenn ich trotzdem noch einmal auf sie zurückkomme, so ist hierfür lediglich die Tatsache maßgebend, dab die Vertreter der Industrie die Arbeit des Herm Evers noch immer zu ihren Gunsten verwerten, und daß der Autor selbst, anstatt auch nur den leisesten Versuch zu einer Erklärung oder Berichtigung seiner seltsamen Ergebnisse zu machen, den vielfachen Angriffen in der Fachpresse gegenüber ein vollkommenes Stillschweigen beobachtet. Vor allem aber erscheint eine nochmalige Besprechung

1) Zeitschr. öffentl. Chem. 1904, 10, 319.

2) Diese Zeitschrift 1904, 8, 538.

3) Diese Zeitschrift 1904, 8, 548 .

$\left.{ }^{4}\right)$ Diese Zeitschrift 1904, 8, 657.

5) Diese Zeitschrift 1905, 9, 141.

6) Zeitschr. offentl. Chem. 1904, 10, 406; diese Zeitschrift 1905, 9, 611.

7) Zeitschr. öffentl. Chem, 1904, 10, 480. 
der Alkalitäts-Frage deshalb geboten, weil Herr Dr. Evers auch jetzt noch fortfährt, in gerichtlichen Gutachten die von Spaeth gesehaffene Grundlage der Himbeersyrup-Beurteilung als falsch und durch seine eigene Arbeit widerlegt hinzustellen. So finden sich z. B. in seinem, am 2. Februar 1905 für eine Verhandlung vor der hiesigen Berufungskammer erstatteten Gutachten, folgende Sätze:

„Im Jahre 1901 sind von dem Erlanger Chemiker Dr. Spaeth Normen für die Beurteilung von Himbeersyrup aufgestellt worden. Dr. Spaeth fordert für reinen Himbeersyrup einen Aschengehalt von mindestens $0,2 \%$ und eine Alkalität der Asche von mindestens $2 \mathrm{cem}$ N.-Süure .... Eine Verfälsehung im Sinne des Fruchtsafthandels würde nur dann vorliegen, wenn der Fruchtsaft durch eine erhebliche Menge Wasser verdünnt worden wäre. Als eine solche kann aber die Verdünnung mit 10\% Wasser nicht angesehen werden. Durch den Zusatz von $10 \%$ wird auch der Syrup in seiner prozentualen Zusammensetzung nicht so stark verändert, dał die Gehaltsziffern für Zucker und Rohsaft aukerhalb der Grenzen fallen, welche für reinen Arzneihimbeersyrup erfahrngssgemäß angenommen werden. Ich habe im verflossenen Jahre zahlreiche sejbstbereitete Himbeersyrupe bezw. Himbeerrohsäte untersucht, die sich bezl. des Aschengehaltes und der Alkalität der Asche wesentlich anders verhielten, als Dr. Spaeth angegeben hat. Aschengehalt und Alkalitat sind oft ganz bedeutend geringer, und die Grenzen so weit auseinandergehend, $d a ß s$ die Bedeutung der Prüfungsmethode von Dr. Spaeth wesentlich eingeschränkt werden muß. 10 selbst $20 \%$ und mehr Wasserzusatz lassen sich chemisch mit Sicherheit nicht feststellen. Auferdem leidet die Prüfungsmethode an sich an solchen Mängeln, daßs bei gleichem Prüfungsobjekt der Befund mehrerer, selbst analysenfester Chemiker meist erheblich verschieden ausfällt . . . . . . Der Alkohol verschlechtert aber den Syrup noch, da ein alkoholfreier Fruchtsaft als gesunder gilt, wie ein bis zn $20 \%$ gespriteter, während durch den Wasserzusatz von $10 \%$ unter Umständen eine Verbesserung erzielt wird, indem ein zu hoher Fruchtsäuregehalt reduziert und dadurch eine zu weit gehende Inversion des Rohrzuckers vermieden wird, die hăufig mit einer sehr lästigen Ausscheidung von Dextrose verbunden ist. Was den Zusatz von Salicylsäure betrifft, so ist hervorzuheben, daßs Salicylsăure ein natürlicher Bestandteil vieler Frïchte, auch der Himbeeren ist, daß also die Natur selbst diese Substanz als Konservierungsmittel gewählt hat ete. ..."

Ich muß es mir versagen, näher auf die erstaunliche Entdeckung einzugehen, daß ein Zusatz von Wasser den Himbeersyrup verbessere, ,weil dadurch ein zu hoher Fruchtsäuregehalt reduziert wird", obwohl es nahe läge, diese Methode im Hinblick auf ihre Verwendbarkeit zur Verbesserung des sehr sauren Citronensaftes, des Bieres und anderer Genußmittel einer Kritik zu unterziehen.

Ich übergehe auch die kühne Schlußfolgerung, daß die in Früchten aufgefundenen minimalen Salicylsäurespuren von der Natur selbst als Konservierungsmittel auserwählt worden seien; eine Idee, die sehr an das von Du Bois-Reymond geprägte berühmte Wort von der ,anscheinend absichtsvoll zweckmäßigen Einrichtung der Natur" anklingt.

Vielmehr will ich mich lediglich auf den Versuch beschränken, noch einmal die Richtigkeit der Spaeth'schen Untersuchungen und damit e contrario das Irrige der entgegengesetzten Beobachtungen des Herrn Dr. Evers darzutun.

Wie Spaeth, Juckenack u. a. habe auch ich schon im vorigen Jabre gleich nach dem Erseheinen der Evers'schen Veröffentlichung darauf hingewiesen ${ }^{1}$ ), då

1) Diese Zeitschrift 1904, 8, 544 . 
das von diesem gefundene Verhältnis zwischen der Menge der Mineralstoffe und ihrer Alkalität zu allen früheren Erfahrungen in Widerspruch steht, indem Evers die Alkalität, ausgedrückt in ccm N.Säure, nicht wie die übrigen Autoren 10-15-mal, sondern nur elwa 5 -mal so hoch wie den Aschengehalt findet. Ich habe dann weiter versucht die Unmöglichkeit eines derartigen Verhältuisses aus der quantitativen $\mathbf{Z u}$ sammensetzung der Mineralstoffe abzuleiten, ohne mir allerdings zu verhehlen, daß die in sehr geringer Zahl vorhandenen Analysen von Himbeersaftaschen für dieses Vorhaben keine hinreichend sichere Grundlage darboten. Und doch schien mir hierin die einzige Möglichkeit einer exakten Beweisführung zu liegen, da selbst die größte Zahl entgegengesetzter Befunde die Unrichtigkeit der Evers'schen Ergebnisse nur immer wahrscheinlicher machen, aber nicht mathematisch sicher dartun kann. Zwar dürfte Evers seine ursprüngliche Erklärung der Spaeth'schen Befunde, nämlich daßo dessen Himbeersäfte ganz abnorm zusammengesetzt gewesen seien, inzwischen als unbaltbar erkannt haben, seitdem die Untersuchungen aller vorgenannten Fachgenossen für Beeren der verschiedensten Herkunft aus Bayern, Brandenburg, Sachsen und Thüringen, ja selbst den Niederlanden zu den gleichen Werten führten, aber er könnte doch immerhin noch behaupten, daß seine sämtlichen Gegner die Alkalität falsch bestimmt hätten, und daß nur seine eigenen Zahlen richtig seien. Dieser zwar unwahrscheinliche, aber immerhin mögliche Einwand kann nur aus der Zusammensetzung der Mineralstoffe widerlegt werden, weil die Alkalität eine einfache Funktion der Aschenbestandteile ist, und ich habe daher zur Ausfüllung der vorhandenen Lücke die Aschenanalyse einer größeren Anzahl yon Fruchtsäften ausgeführt und dazu neben dem Himbeersaft auch Citronensaft, Kirschsaft, Johannisbeersaft und Erdbeersaft in den Kreis der Untersuchung mit einbezogen.

Die Herstellung der Asche wurde in der üblichen Weise, und zwar vorsichtshalber, um eine Verunreinigung durch den Schwefelgehalt des Leuchtgases sicher zu vermeiden, mit Hilfe des Spiritusbrenners ausgeführt, wenngleich ich Lührig darin vollkommen beistimme, daß man für die Zwecke der Praxis ruhig auf einer durchlochten Asbestplatte mit der Gasflamme veraschen kann, ohne einen Fehler befürchten zu müssen. Die Bestimmung der Alkalität erfolgte nach dem Übersätuigen mit Schwefelsäure und dem Verjagen des Kohlendioxyds durch Rückitration mit 1/10 N.Kalilauge unter Verwendung von Phenolphtalein als Indikator.

Zur Abscheidung der Kieselsäure wurde eine gewogene Menge der Mineralstoffe in einer Porzellanschale ${ }^{x}$ mit Salzsäure mehrmals eingedampft, und der beim Aufnehmen mit Wasser ungelöst bleibende Rückstand abfiltriert und gewogen. Von dem auf ein bestimmtes Volum aufgefüllten Filtrate diente ein Teil zur Bestimmung der Schwefelsäure und der Alkalien. In einem anderen wurden zunächst durch Kochen der neutralisierten und mit Natriumacetat versetzten Lösung Eisen und Tonerde als Phosphate, und im Filtrate davon Kalk und Magnesia gefällt. Für die Bestimmung der Kohlensäure (im Geißler'schen Apparate), der Phosphorsäure (Molybdänmethode) und endlich des Chlors wurden besondere Portionen Asche benutzt. In einigen Fällen habe ich auch nach dem v. Knorre'schen Verfahren den Mangangehalt ermittelt, sehe aber einstweilen von einer Mitteilung der erhaltenen Werte $(0,2-0,3 \%)$ als für den vorliegenden $Z$ weck belanglos ab, um vielleicht später an der Hand eines größeren

i) Die direkte Behandlung der Asche in der Platinschale verbietet sich, weil der meist nicht unbeträchtliche Mangangehalt eine Chlorentwickelung im Gefolge hat. 
Zahlenmaterials auf diesen für manche Fruchtsäfte charakteristischen Bestandteil zurückzukommen.

In der nachstehenden Tabelle sind zunächst die für alle untersuchten Aschen erlangten Werte eingetragen, und außerdem habe ich zur Vervollständigung drei von Farnsteiner ausgeführte Analysen, nämlich eine von Citronensaft- ${ }^{1}$ ) und zwei von Apfelsinensaftaschen ${ }^{2}$ ) hinzugestellt.

Tabelle I.

\begin{tabular}{|c|c|c|c|c|c|c|c|c|c|c|c|}
\hline No. & $\begin{array}{c}\text { Bezeichnung } \\
\text { des Fruchtsaftes }\end{array}$ & $\begin{array}{c}\text { Kali } \\
\left(\mathrm{K}_{2} \mathrm{O}\right) \\
\\
\alpha_{0}\end{array}$ & $\begin{array}{l}\text { Natron } \\
\left(\mathrm{Na}_{2} \mathrm{O}\right)\end{array}$ & $\begin{array}{l}\text { Kalk } \\
(\mathrm{Ca} \mathrm{O})\end{array}$ & $\begin{array}{l}\text { Mag- } \\
\text { nesia } \\
\text { (MgO) } \\
\%_{0}\end{array}$ & 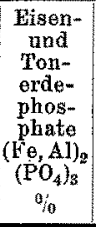 & $\begin{array}{c}\text { Kiesei- } \\
\text { süure } \\
\left(\mathrm{SiO}_{2}\right) \\
\\
\%_{0}\end{array}$ & $\begin{array}{c}\text { Phos- } \\
\text { phor- } \\
\text { säure } \\
\left(\mathrm{P}_{2} \mathrm{O}_{5}\right) \\
\\
\mathbb{0}_{0} / 0\end{array}$ & $\begin{array}{c}\text { Sehwe- } \\
\text { felsäure } \\
\left(\mathrm{SO}_{3}\right) \\
\\
\%_{\%}\end{array}$ & $\begin{array}{l}\text { Chlor } \\
\text { (Cl) } \\
\%\end{array}$ & $\begin{array}{c}\text { Kohlen- } \\
\text { säure } \\
\left(\mathrm{CO}_{2}\right) \\
\\
\%_{\%}\end{array}$ \\
\hline 1 & Himbeersaft & 49,70 & 1,05 & 7,51 & 5,67 & 0,56 & 0,58 & 8,87 & 4,03 & 1,26 & 25,32 \\
\hline 2 & & 50,61 & 1,10 & 8,26 & 5,01 & 1,42 & 0,27 & 6,25 & 3,10 & 0,86 & 28,60 \\
\hline 3 & & 48,03 & 1,00 & 9,68 & 4,92 & 0,86 & 0,43 & 6,19 & 2,61 & 1,82 & 27,63 \\
\hline 4 & & \multicolumn{2}{|c|}{51,23} & 7,89 & 5,80 & 0,38 & 0,36 & 7,50 & 1,20 & 0,63 & 29,02 \\
\hline 5 & , mit Naehpresse & 47,01 & 1,70 & 9,48 & 5,93 & 1,02 & 0,26 & 5,03 & 2,64 & 0,92 & 28,74 \\
\hline 6 & Citronensaft & 50,01 & 2,22 & 9,25 & 3,27 & 3,00 & 1,11 & 5,32 & 2,12 & 0,27 & 28,60 \\
\hline 7 & 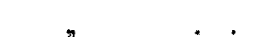 & 46,07 & 2,00 & 7,90 & 4,93 & 3,41 & 0,66 & 3,40 & 1,83 & 1,20 & 29,59 \\
\hline 8 & & 48,30 & 1,96 & 8,14 & 4,06 & 2,48 & 0.86 & 4,38 & 1,90 & 0,80 & 29,04 \\
\hline 9 & & 46,80 & 3,00 & 9,00 & 4,44 & 2,35 & 1,00 & 6,27 & 2,05 & 0,93 & 28,01 \\
\hline 10 & (Farnsteiner) & 43,50 & 3,45 & 9,28 & 5,57 & 1,59 & - & 7,22 & 2,12 & 1,59 & 28,65 \\
\hline 11 & Kirschsaft & 50,26 & 5,10 & 5,00 & 4,30 & 3,14 & 0,56 & 7,30 & 2,38 & 0,11 & 28,00 \\
\hline 12 & & \multirow{2}{*}{\multicolumn{2}{|c|}{$\begin{array}{l}52,38 \\
56,38\end{array}$}} & 5,68 & 2,33 & 3,83 & 1,63 & 8,66 & 1,17 & 0,21 & 25,81 \\
\hline 13 & $"$ & & & 4,96 & 2,14 & 3,45 & 0,96 & 8,49 & 1,19 & 0,96 & 26,00 \\
\hline 14 & \multirow{2}{*}{$\underset{\text { sinensaft }}{\text { Apfel- }}\} \begin{array}{c}\text { (Farn- } \\
\text { steiner) }\end{array}$} & 48,56 & 2,89 & 6,01 & 4,57 & 0,96 & - & 6,25 & 1,44 & 1,68 & 29,81 \\
\hline 15 & & 50,66 & 1,69 & 7,88 & 3,94 & 1,88 & - & 5,07 & 3,94 & 0,56 & 29,64 \\
\hline 16 & \multirow{2}{*}{$\begin{array}{c}\text { Johannisbeersaft. } \\
n\end{array}$} & \multirow{2}{*}{\multicolumn{2}{|c|}{$\begin{array}{l}58,70 \\
52,20\end{array}$}} & 4,26 & 4,31 & 0,38 & 0,11 & 15,64 & 1,28 & 0,20 & 20,10 \\
\hline 17 & & & & 5,39 & 2,59 & 2,42 & 1,04 & 5,64 & 1,64 & 0,24 & 28,90 \\
\hline 18 & Erdbeersaft & \multicolumn{2}{|c|}{42,50} & 12,05 & 4,10 & 0,69 & 0,63 & 8,56 & 1,00 & 0,81 & 27,05 \\
\hline
\end{tabular}

Um aus der analytisch ermittelten Zusammensetzung der Asche die Alkalität gegen Phenolphtalein zu erfahren, rechnet man zweckmäfig zunächst die sämtlichen Basen: Kalium, Natrium, Calcium und Magnesium auf das entsprechende Äquivalent Kaliumoxyd um. Von der erbaltenen Summe subtrahiert man die Kalimengen, welehe von der Schwefelsäure, dem Chlor und der Phosphorsäure zur Bildung der gegen Phenolphtalein neutralen Verbindungen $\mathrm{K}_{2} \mathrm{SO}_{4}$, $\mathrm{KCl}$ und $\mathrm{K}_{2} \mathrm{HPO}_{4}$ verbraucht werden, und erfährt dann aus dem verbleibenden Rest die Alkalität der Asche.

1) Diese Zeitschrift $1909,6,15$.

2) Diese Zeitsehrift 1904, 9, 603 . 
Zur völligen Klarstellung meines Gedankenganges gestatte ich mir die Rechnung an einem praktischen Beispiele, dem Himbeersaft No. 1, durchzuführen:

Die Asche enthält 49,7\% $\mathrm{K}_{2} \mathrm{O} ; 1,05 \% \mathrm{Na}_{2} \mathrm{O} ; 7,51 \% \mathrm{CaO} ; 5,67 \% \mathrm{MgO}$. Unter Zugrundelegung der internationalen Atomgewichte $(0=16)$ entsprechen

$$
\begin{aligned}
& 62,1 \text { g Na}{ }_{2} \mathrm{O}=94,3 \text { g K}{ }_{2} \mathrm{O} \text {; also } 1,05 \% \mathrm{Na}_{2} \mathrm{O}=1,57 \% \mathrm{~K}_{2} \mathrm{O} \\
& 56,0, \mathrm{CaO}=94,3, \mathrm{~K}_{2} \mathrm{O} \text {; also } 7,51, \mathrm{CaO}==12,66 \text {, n } \\
& 40,36, \mathrm{MgO}=94,3, \mathrm{~K}_{2} \mathrm{O} \text {; also } 5,67 \text {, } \mathrm{MgO}=13,25 \text {, , } \\
& \frac{\text { dazu gefunden } \cdot . \cdot .49,70 \%}{\text { gibt als Äquivalent der Basen } 77,18 \% \mathrm{~K}_{2} \mathrm{O}}
\end{aligned}
$$

Das Äquivalent der Säuren erhält man in folgender Weise: Die Asche enthält 4,03\% $\mathrm{SO}_{3} ; 1,26 \%$ Cl und $8,87 \% \mathrm{P}_{2} \mathrm{O}_{5}$.

Nach der Gleichung: $\mathrm{SO}_{3}+\mathrm{K}_{2} \mathrm{O}=\mathrm{K}_{2} \mathrm{SO}_{4}$ entsprechen

$$
80,06 \mathrm{~g} \mathrm{SO}_{3}=94,3 \mathrm{gK}_{2} \mathrm{O} \text {; also } 4,03 \% \mathrm{SO}_{3}=4,75 \% \mathrm{~K}_{2} \mathrm{O} \text {. }
$$

Nach der Gleichung: $2 \mathrm{HCl}+\mathrm{K}_{2} \mathrm{O}=2 \mathrm{KCl}+\mathrm{H}_{2} \mathrm{O}$ entsprechen

$70,9 \mathrm{~g} \mathrm{Cl}=94,3 \mathrm{~g} \mathrm{~K}_{2} \mathrm{O}$; also $1,26 \% \mathrm{Cl}=1,68 \% \mathrm{~K}_{2} \mathrm{O}$.

Nach der Gleichung: $\mathrm{P}_{2} \mathrm{O}_{5}+2 \mathrm{~K}_{2} \mathrm{O}+\mathrm{H}_{2} \mathrm{O}=2 \mathrm{~K}_{2} \mathrm{HPO}_{4}$ entsprechen

$$
\begin{aligned}
& 71,0 \text { g } \mathrm{P}_{2} \mathrm{O}_{5}=94,3 \mathrm{~g} \mathrm{~K}_{2} \mathrm{O} \text {; also 8,87\% } \mathrm{P}_{2} \mathrm{O}_{5}=11,78 \% \quad \mathrm{~K}_{2} \mathrm{O} \text {; } \\
& \text { also Äquivalent der Säuren }=18,21 \% \mathrm{~K}_{2} \mathrm{O} \text {. }
\end{aligned}
$$

Die Differenz beider Äquivalente 77,18-18,21 =58,97 ergibt die Menge des freien, bezw. in der Asche an Kohlensäure gebundenen Kaliumoxyds, aus dem sich nach dem Ansatz $47,15 \mathrm{~g} \mathrm{~K}_{2} \mathrm{O}=11 \mathrm{~N}$. Säure die Alkalitat $\mathrm{zu} \frac{58,97 \times 1000}{47,15}=1251$ berechnet; d. h. $100 \mathrm{~g}$ Asche haben eine Alkalität von 1251; das Verhältnis von Asche zu Alkalität ist 1:12,51.

Der einfachere Weg, die Alkalität aus dem analytisch ermittelten Kohlensäuregehalte allein zu berechnen, führt zu unrichtigen Werten, weil in der Asche neben den Karbonaten nicht das gegen Phenolphtalein neutral reagierende Dikaliumphosphat, $\mathrm{K}_{2} \mathrm{HPO}_{4}$, sondern Trikaliumphosphat $\mathrm{K}_{3} \mathrm{PO}_{4}$ enthalten ist. Es muß daher zu dem Äquivalent der Kohlensäure noch das des Trikaliumphosphates hinzugenommen werden, im Sinne der Gleichung:

$$
\mathrm{K}_{3} \mathrm{PO}_{4}+\mathrm{HCl}=\mathrm{K}_{2} \mathrm{HPO}_{4}+\mathrm{HCl}
$$

d. h. für je $71 \mathrm{~g} \mathrm{P}_{2} \mathrm{O}_{5}$ ist $1 \mathrm{l}$ N.-Säure zu addieren.

Diese Rechnung gestaltet sich bei dem konkreten Beispiel, dem Himbeersaft No. 1 folgendermaßen:

Gefunden wurden 25,32\% $\mathrm{CO}_{2}$ und 8,87\% $\mathrm{P}_{2} \mathrm{O}_{5}$.

$44 \mathrm{~g} \mathrm{CO}_{2}=2000 \mathrm{ccm}$ N.-Säure; $25,32 \% \quad \mathrm{CO}_{2}=1151 \mathrm{ccm}$ N.-Säure

$71 \mathrm{~g} \mathrm{P}_{2} \mathrm{O}_{5}=1000 \quad, \quad, \quad ; 8,87 \% \mathrm{P}_{2} \mathrm{O}_{5}=125, ", \quad$,

$$
\text { Alkalität }=1276 \text {. }
$$

Ganz genau wird diese Berechnung übrigens auch unter Berücksichtigung des Phosphorsäureäquivalentes niemals, weil bekanntlich nach $\mathrm{Woy}^{1}$ ) ein Teil der Phosphorsäure beim Glühen in Pyrophosphat übergeht, und für diesen Fehler keine Korrektur angebracht werden kann.

Führt man die soeben angegebene Rechnung für die sämtlichen in Tabelle I angeführten Aschenanalysen aus, so erlangt man folgende Werte:

1) Chem.-Ztg. 1897, 21, 471. 
Tabelle II

\begin{tabular}{|c|c|c|c|c|c|c|c|}
\hline \multirow{3}{*}{ No. } & \multirow{3}{*}{$\begin{array}{c}\text { Bezeichnung } \\
\text { des Fruchtsaftes }\end{array}$} & \multirow{3}{*}{$\begin{array}{c}\text { Mineral- } \\
\text { stoffe } \\
\text { im Saft } \\
\\
0 \%\end{array}$} & \multicolumn{2}{|c|}{ Alkalität } & \multicolumn{3}{|c|}{$\begin{array}{l}\text { Verbältnis von Asche }(\%) \\
\text { zu Alkalität (ecm N.-Süure) }\end{array}$} \\
\hline & & & \multirow{2}{*}{$\begin{array}{l}\text { gefunden } \\
\text { cem } N \text {.- } \\
\text { Säure }\end{array}$} & \multirow{2}{*}{$\begin{array}{c}\text { berechnet } \\
\text { ans Basen } \\
\text { und Säuren } \\
\text { cem N.- } \\
\text { Säure }\end{array}$} & \multirow[b]{2}{*}{$\begin{array}{l}\text { gefunden } \\
=1\end{array}$} & \multicolumn{2}{|c|}{ berechnet aus } \\
\hline & & & & & & $\begin{array}{c}\text { Basen und } \\
\text { Sämren } \\
=1:\end{array}$ & $\begin{array}{l}\text { Kohlen- } \\
\text { säure allein } \\
\quad=1 \text { : }\end{array}$ \\
\hline 1 & Himbeersaft & 0,654 & 8,14 & 8,18 & 12,4 & 12,5 & 11,5 \\
\hline 2 & . . & 0,702 & 9,30 & 9,67 & 13,2 & 13,8 & 13,0 \\
\hline 3 & & 0,612 & 8,20 & 8,26 & 18,5 & 18,5 & 12,6 \\
\hline 4 & . . & 0,679 & 9,28 & 9,51 & 13,7 & 14,0 & 13,2 \\
\hline 5 & mit Nachpresse & 0,416 & 6,07 & 6,08 & 14,6 & 14,6 & 13,1 \\
\hline 6 & Citronensaft . & 0,526 & 7,30 & 7,43 & 13,9 & 14,1 & 13,0 \\
\hline 7 & \# & 0,514 & 7,20 & 7,13 & 14,0 & 13,7 & 13,4 \\
\hline 8 & " & 0,482 & 6,60 & 6,68 & 13,7 & 13,9 & 13,2 \\
\hline 9 & . . . . . & 0,450 & 6,10 & 6,20 & 13,6 & 13,7 & 12,7 \\
\hline 10 & $n \quad$ (Farnsteiner). & 0,377 & 4,91 & 5,04 & 13,0 & 13,4 & 13,0 \\
\hline 11 & Kirschsaft . . . . . . & 0,540 & 7,40 & 7,39 & 13,7 & 13,7 & 12,8 \\
\hline 12 & . . . . . & 0,600 & 7,02 & 6,89 & 11,7 & 11,5 & 11,7 \\
\hline 13 & $n \quad \cdot \cdot \cdot \cdot \cdot \cdot \cdot \cdot$ & 0,481 & 5,45 & 5,49 & 11,3 & 11,4 & 11,8 \\
\hline 14 & Apfelsinensaft (Farnsteiner) & 0,416 & 5,62 & 5,37 & 13,5 & 12,9 & 13,5 \\
\hline 15 & $\pi \quad 1 \quad n$ & 0,583 & 7,20 & 7,20 & 13,5 & 13,5 & 13,5 \\
\hline 16 & Johannisbeersaft . & 0,586 & 5,60 & 6,02 & 9,5 & 10,3 & 9,1 \\
\hline 17 & . . . & 0,602 & 7,62 & 7,36 & 12,6 & 12,2 & 13,1 \\
\hline 18 & Erdbeersaft. . . . , & 0,514 & 6,42 & 6,48 & 12,5 & 12,6 & 12,3 \\
\hline
\end{tabular}

Diese Tabelle lehrt zunächst, daß bei allen 18 Fruchtsaftaschen, und zwar sowohl bei den 3 von Farnsteiner, wie den 15 von mir untersuchten, die gefundene Alkalität mit der aus der Aschenanalyse berechneten innerhalb der unvermeidlichen Fehlergrenze übereinstimmt, und beweist sonach in unanfechtbarer Weise, daß das von mir benutzte Verfahren der Alkalitätsbestimmung zu richtigen Werten führt. Die Tabelle bestätigt aber auch, in Obereinstimmung mit meinen früheren Angaben, dab das Verbältnis der Mineralstoffe zu ihrer Alkalität zwischen $1: 10$ und $1: 15$ liegt, und beweist damit außerdem, dah alle die nach dem gleichen Verfahren von Spaeth, Juckenack, Lührig und den übrigen Autoren gefundenen Alkalitätswerte richtig sein müssen. Wenn also irgend jemand abnorme Säfte in Händen gehabt hat, so ist dies, darüber kann kein Zweifel aufkommen, nicht $S p a$ eth, sondern höchstens Herr Dr. Evers gewesen.

Es läBt sich aber auf Grund der mitgeteilten Aschenanalysen noch weiter dartun, daB die Evers'schen Zahlen selbst an ganz abnormen Säften gar nicht gewonnen sein können, sondern daf das von ihm mitgeteilte Verbältnis von Mineralstoffen zu Alkalitat eine analytische Unmöglichkeit ist. Die Alkalität einer Fruchtsaftasche wird um so niedriger ausfallen, je höher der Gehalt an Schwefelsäure und Chlor ist, und je mehr der Kalk 
und die Magnesia den Alkalien gegenüber zurücktreten, weil ihre Alkalität wesentlich höher als die des Kalis ist. Machen wir also einmal zugunsten der Evers'schen Befunde, d. h. zur Ableitung einer Asche mit möglichst niedriger Alkalität die Annahme, wir hätten eine Himbeersaftasche mit recht viel Schwefelsäure und Chlor, hingegen recht wenig Kalk und Magnesia.

Nach allen Erfahrungen wird man einen Schwefelsäuregehalt von $4 \%$ und einen Chlorgehalt von $2 \%$ als anßergewöhnlich hoch bezeichnen müssen. Setzen wir daher diese Zahlen ein und nebmen dazu weiter den niedrigsten von uns gefundenen Wert für Kalk mit $4,26^{\circ}, 0$ und für Magnesia mit 2,14\%, ferner für Eisen, Mangan und Kieselsäure zusammen noch rund $2 \%$, so erhalten wir als Summe aller dieser Bestandteile 14,40, bezw. nach Abzug des Sauerstoff-Äquivalentes für $2 \%$ Chlor die Zahl 13,95\%. Dazu müssen wir noch wegen des Überschusses der Basen iüber die Sänren eine gewisse Menge Kohlensäure hinzurechnen, welche wir in folgender Weise finden:

$56 \mathrm{~g} \mathrm{CaO}$ entsprechen 40,36 g MgO; die vorhandenen 4,26\% CaO entsprechen also $3,07 \%$ MgO. Werden hierzu die analytisch gefnndenen $2,14 \%$ MgO addiert, so ergibt sich als Äquivalent der vorhandenen Basen 5,31\% MgO.

Weiter entsprechen

$80,0 \mathrm{~g} \mathrm{SO}_{3}=40,36 \mathrm{~g} \mathrm{MgO} ; 4 \% \mathrm{SO}_{3}$ demnach $2,018 \% \mathrm{MgO}$;

70,$9 ; \mathrm{Cl}=40,36 \mathrm{~g} \mathrm{MgO} ; 2 \% \mathrm{Cl}, 1,138$,

Das Äquivalent der vorhandenen Säuren, in Magnesia ausgedrückt, beträgt dann die Summe beider: $3,156 \%$. Der Übersehuß der Gesamtbasen über die von Sâure gebundenen 5,21 minus $3,156=2,054 \% \mathrm{MgO}$ - tritt als Alkalität der vorgenannten Bestandteile in die Erscheinung und entspricht nach dem Ansatze

$40,36 \mathrm{~g} \mathrm{MgO}=2000 \mathrm{~cm} \mathrm{~N}$.-Säure $; 2,054 \% \mathrm{MgO}=101,8 \mathrm{~cm}$ einer Alkalität von $101,8 \mathrm{ccm} \mathrm{N}$.-Säure.

Die nicht durch Schwefelsäure und Chlor abgesättigțen alkalischen Erden müssen in der Asche als Karbonate zugegen sein, und zwar betrâgt die Menge der in ihnen enthaltenen Kohlensäure $2,054 \frac{\mathrm{CO}_{2}}{\mathrm{MgO}}=2,054 \cdot \frac{44}{40,36}=2,24 \% \mathrm{CO}_{2}$.

Demnach erhalten wir als Summe der in dieser Asche enthaltenen Sulfate, Chloride und Karbonate des Calciums und Magnesiums, ferner des Eisens, Mangans und der Kieselsäure die Zahl $\mathrm{A}=16,19 \%$. Die übrigen $83,81 \%$ der Mineralstoffe bestehen aus Kaliumkarbonat und Trikaliumphosphat. Nennen wir die Menge des ersteren $\left(\mathrm{K}_{2} \mathrm{CO}_{3}\right) \mathbf{x}$, diejenige des letzteren $\left(\mathrm{K}_{3} \mathrm{PO}_{4}\right)$ y, so ergibt sich als erste Gleichung

$$
\mathrm{x}+\mathrm{y}=83,81 \text {. }
$$

Um die Zusammensetzung einer Asche von der Gesamtalkalität 540, entsprechend dem Mittelwert der Evers'schen Versuche, zu berechnen, leiten wir die zweite Gleichung aus der Alkalität der Einzelbestandteile in folgender Weise ab: Die Alkalität der Summe aller Aschenbestandteile 540 vermindert um die Alkalität der Zahl $\mathrm{A}=101,8$ ergibt für die Alkalität des Kaliumkarbonates und -Phosphates 438,2.

Nun verbrauchen nach den Gleichnngen

$$
\begin{aligned}
& \mathrm{K}_{2} \mathrm{CO}_{3}+2 \mathrm{HCl}=2 \mathrm{KCl}+\mathrm{H}_{2} \mathrm{O}+\mathrm{CO}_{2} ; \text { und } \mathrm{K}_{3} \mathrm{PO}_{4}+\mathrm{HCl}=\mathrm{K}_{2} \mathrm{HPO}_{4}+\mathrm{KCl} \\
& 138,30 \mathrm{~g} \mathrm{~K}_{2} \mathrm{CO}_{3}=21 \mathrm{~N} \text {. Säure; } \mathrm{x} \mathrm{g} \mathrm{K}_{2} \mathrm{CO}_{3} \text { also } \mathrm{x} \cdot \frac{2000}{138,3} \mathrm{ccm} \\
& 212,45 \mathrm{~g} \mathrm{~K}_{3} \mathrm{PO}_{4}=11 \mathrm{~N} \text {.-Säure; y } \mathrm{g} \mathrm{K} \mathrm{KO}_{4} \text { also y } \cdot \frac{1000}{212,45} \mathrm{ccm} \text {. }
\end{aligned}
$$

Die zweite Gleichung lautet demnach:

$$
\mathrm{x} \cdot \frac{2000}{138,3}+\mathrm{y} \cdot \frac{1000}{212,45}=438,2
$$

Aus beiden Gleichungen berechnet sich:

N. 05. 


$$
\begin{aligned}
& x=4,45 ; \quad y=79,36 \\
& \text { d. h. die Asche enthält } 4,45 \% \mathrm{~K}_{2} \mathrm{CO}_{3} \\
& \text { und } 79,36 \% \mathrm{~K}_{3} \mathrm{PO}_{4} \\
& \text { oder } 55,87 \% \quad \mathrm{~K}_{2} \mathrm{O} ; 26,52 \% \mathrm{P}_{2} \mathrm{O}_{5} ; 1,42 \% \mathrm{CO}_{2} \text {. }
\end{aligned}
$$

Unter Hinzurechnung des Wertes A hat also eine Asche mit der Alkalität 540 folgende Zusammensetzung:

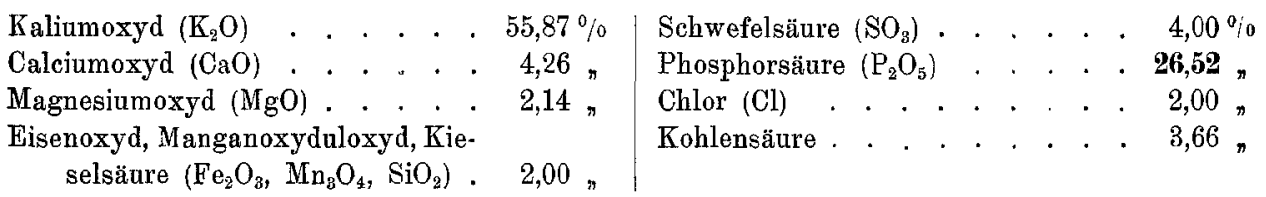

Bieten schon diese Werte einen merkwürdigen Anblick dar, so werden die Ergebnisse noch seltsamer, wenn man die gleiche Rechnung für die niedrigste von Evers mitgeteilte Alkalität 490 ausfïhrt.

Dann ergibt sich aus den Gleichungen

$$
\begin{gathered}
x+y=83,81 \\
x \cdot \frac{2000}{138,3}+y \cdot \frac{1000}{212,45}=490-101,8 \\
x=-0,68 \% \mathrm{~K}_{2} \mathrm{CO}_{3} ; y=84,49 \% \mathrm{~K}_{3} \mathrm{PO}_{4} .
\end{gathered}
$$

Der negative Wert für $\mathrm{x}$ bedeutet, daß3 bereits unter Zurechnung der für $\mathrm{K}_{3} \mathrm{PO}_{4}$ allein gefundenen Zahl sich eine Alkalität von mehr als 490 ergibt. Es muß also das im Kaliumkarbonat enthaltene Kaliumoxyd von dem Kali des Phosphates and die entsprechende Menge Kohlensäure von der an die alkalischen Frden gebundenen Kohlensäure in Abzug gebracht werden, damit die frei werdende Phosphorsäure mit dem überschüssigen Calcium- oder Magnesiumoxyd zusammentreten kann.

Nun enthalten die $84,49 \% \mathrm{~K}_{3} \mathrm{PO}_{4} \ldots 56,25 \% \mathrm{~K}_{2} \mathrm{O}$ und $28,24 \% \mathrm{P}_{2} \mathrm{O}_{5}$

$$
\text { die } 0,68 \% \mathrm{~K}_{2} \mathrm{CO}_{3} \ldots 0,46 \% \mathrm{~K}_{2} \mathrm{O} \text { und } 0,26 \% \mathrm{CO}_{2} \text {, }
$$

es verbleiben also nach der Subtraktion: $55,79 \% \quad \mathrm{~K}_{2} \mathrm{O}$ und $2,24-0,26=1,98 \% \mathrm{CO}_{2}$ und wir erhalten für die Asche von der Alkalität 490 nachstehende Zusammensetzung:

\begin{tabular}{|c|c|c|c|c|c|c|c|c|}
\hline & Alk & lität & & & & & & lität \\
\hline & 540 & 490 & & & & & $\mathbf{5 4 0}$ & 490 \\
\hline & $0 \%$ & $0 / 0$ & & & & & $\%$ & $\%$ \\
\hline Kaliumoxyd $\left(\mathrm{K}_{\mathbf{z}} \mathrm{O}\right)$ & 45,71 & 45,63 & Schwefelsäure $\left(\mathrm{SO}_{3}\right)$ & . & & & 4,0 & 4 \\
\hline Calciumoxyd $(\mathrm{CaO})$ & 8,56 & 8,56 & Phosphorsäure $\left(\mathrm{P}_{2} \mathrm{O}_{5}\right)$ & . & . & & $\mathbf{3 0}, \mathbf{1}$ & $32, \mathrm{C}$ \\
\hline Magnesiumoxyd $(\mathrm{MgO})$ & 5,47 & 5,47 & Chlor $(\mathrm{Cl})$. . . . & . & . & & 2,0 & 2,0 \\
\hline Eisenoxyd, Manganoxyduloxyd, & & & Kohlensäure $\left(\mathrm{CO}_{2}\right)$ & & & & 2,5 & \\
\hline Kieselsäure ( $\mathrm{Fe} O \mathrm{M}, \mathrm{O}, \mathrm{SiO}$ ) & 2,00 & 2,00 & & & & & & \\
\hline
\end{tabular}

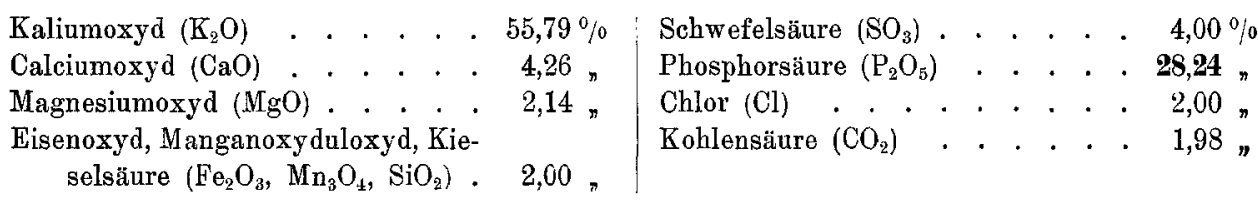

Wenn man endlich unter sonst gleichbleibenden Verhältnissen nicht die für andere Fruchtsäfte gefundenen Minimalwerte an Kalk und Magnesia, sondern den mittleren Kalkund Magnesiagehalt des $\mathrm{Himbeersaftes}$ mit 8,56 bezw. 5,47 einsetzt und dann die Rechnung für die Alkalitäten 540 und 490 ausführt, so erhält man

$$
\begin{aligned}
& \text { im ersten Falle: } x=-21,16 ; \quad y=90,33 \\
& \text { im zweiten Falle: } x=-26,67 ; \quad y=95,84
\end{aligned}
$$

und als Zusammensetzung der Mineralstoffe: 
Man sieht also, daß für das Zustandekommen einer niedrigen Alkalität der Phosphorsäuregehalt der Asche von bestimmendem Einfluß ist und daher vor allem berücksichtigt werden muß. Gerade über diesen Bestandteil besitzen wir aber die meisten Erfahrungen, und ich selbst habe den Phosphorsäuregehalt von mehr als 50 Fruchtsäften ermittelt. Aus diesem reichhaltigen Analysenmaterial ${ }^{1}$ ) geht aber unzweifelhaft hervor, daß der Phosphorsäuregehalt im allgemeinen zwischen 4,6 und $8,5 \%$ liegt und nur in vereinzelten Fällen (bei Walderdbeeren) auf 10-13\% ansteigt, sodaß Werte von $25-32 \%$ als völlig ausgeschlossen bezeichnet werden können. Da es außerdem Fruchtsaftaschen fast ohne eine Spur von Kohlensäure selbstverständlich nicht gibt, so ist hierdurch mit absoluter Sicherheit bewiesen, daßein Alkalitätswert von $4,9-5,4$ ccm N.-Säure eine analytische Unmöglichkeit ist, und daß die von Herrn Dr. Evers angegebenen Zahlen falsch bestimmt worden sind. Es kann daher wohl erwartetwerden, dab diese unrichtigen Befunde in Zukunft nicht weiter zur Grundlage von Gutachten benutzt werden.

Dresden, im Juli 1905.

1) Diese Zeitschrift 1903, 6, 1105 und 1904, 8, 544 und 547.

\title{
Beiträge zur Kenntnis des Sesamöles.
}

\author{
Von
}

\section{H. Sprinkmeyer und H. Wagner.}

\section{Mitteilung aus dem Staatlichen Chemischen Untersuchungsamt für die} Auslandsfleischbeschau zu Goch.

Das aus dem Samen des Sesamstrauches gewonnene ÖI hatte vor Inkrafttreten des Gesetzes, betreffend den Verkehr mit Butter, Käse, Schmalz und (leren Ersatzmitteln vom 15. Juni 1897, nur eine untergeordnetere Stellung in der Reihe der im Großbetrieb hergestellten Speiseöle inne. Seitdem ist der Verbrauch vor allem in der Margarine-Industrie derart gestiegen, daß man heute außer der indischen auch die levantinische und die afrikanische Sesamsaat, die früher zur Herstellung von Speiseöl nicht verwendet wurden, zur Gewinnung heranzieht. Da die Literatur bisher nur wenige Angaben über Sesamöle verschiedener Herkunft aufweist, - die einzige Arbeit, die wir über diesen Gegenstand gefunden haben, ist eine Abhandlung von F. $\mathrm{Utz}^{1}$ ) - so entschlossen wir uns, die wichtigsten chemischen und physikalischen Konstanten der aus den im Handel vorkommenden drei Sesamsaaten, der indischen, der levantinischen und der afrikanischen Saat, gewonnenen Öle festzustellen und die über diesen Gegenstand vorhandenen Literaturangaben einer Nachprüfung zu unterziehen.

Um einerseits einem Irrtum in der Herkunft des Öles möglichst vorzubeugen, und andererseits Öle verschiedener Herstellungsweise zu besitzen, verschafften wir uns die Samen des indisehen, des levantinischen und des afrikanischen Sesamstrauches.

1) Pharm. Ztg. 1900, 45, 490. 